\title{
BMJ Open Smoking cessation using preference- based tools among socially disadvantaged smokers: study protocol for a pragmatic, multicentre randomised controlled trial
}

\author{
Fabienne El-Khoury (1) , ${ }^{1,2}$ Tarik El Aarbaoui, ${ }^{1}$ Mégane Héron, ${ }^{1}$ Gilles Hejblum, ${ }^{3}$ \\ Brigitte Métadieu, ${ }^{4}$ Anne-Laurence Le Faou, ${ }^{5,6}$ Gladys Ibanez (D) ,7,8 \\ Maria Melchior (1D ${ }^{1}$
}

To cite: El-Khoury F, El Aarbaoui T, Héron M, et al. Smoking cessation using preference-based tools among socially disadvantaged smokers: study protocol for a pragmatic, multicentre randomised controlled trial. BMJ Open 2021;11:e048859. doi:10.1136/ bmjopen-2021-048859

- Prepublication history and additional supplemental material for this paper are available online. (http://dx.doi.org/10. 1136/bmjopen-2021-048859).

Received 08 January 2021 Accepted 11 June 2021
Check for updates

(c) Author(s) (or their employer(s)) 2021. Re-use permitted under CC BY-NC. No commercial re-use. See rights and permissions. Published by BMJ.

For numbered affiliations see end of article.

Correspondence to Dr Fabienne El-Khoury; fabienne.khoury@gmail.com

\section{ABSTRACT}

Introduction Many smoking cessation aids such as nicotine replacement treatments and e-cigarettes have been proven effective in aiding smoking cessation attempts. Encouraging smokers with low socioeconomic position (SEP) to choose their smoking aid tool based on their preferences, and giving that tool free of charge, might increase the odds of smoking cessation. This trial examines the effectiveness of the 'STOP' (Sevrage Tabagique à l'aide d'Outils dédiés selon la Préférence: Smoking cessation using preference-based tools), a preference-based smoking cessation intervention for smokers with low SEP.

Methods and analysis The STOP study is a randomised, multicentre, controlled trial (RCT). Smokers with low SEP and wishing to quit will be randomised to either the intervention or the control group (standard care). Participants in the intervention group will be asked to choose between different types of nicotine substitutes (patches, inhalers, gum, tablets, etc) and/or an electronic cigarette which will be delivered free of charge to aid their smoking cessation attempt.

The primary outcome will be smoking abstinence at 6 months after inclusion, defined as self-reported 7-day point prevalence of tobacco abstinence. Secondary outcomes include the total number of days of abstinence at 6 months after inclusion, 7-day point prevalence tobacco abstinence at 1 and 3 months after inclusion and number of relapses.

The study will also include an economic evaluation, and a process evaluation using a mixed methods approach. Ethics and dissemination The study was approved by the 'Île de France II' Institutional Review Board on 8 September 2020 (CPP Île de France II; Ref No: 20.01.31.65528 RIPH2 HPS), and results will be published in a peer-reviewed journal.

Trial registration number NCT04654585.

\section{BACKGROUND}

Nicotine replacement treatments (NRT) and electronic cigarettes (e-cigarettes) have

\section{Strengths and limitations of this study}

- This will be the first randomised controlled trial (RCT) evaluating a preference-based smoking cessation intervention targeted at smokers with low socioeconomic position.

- The study will include an economic evaluation, as well as process evaluation which will allow us to understand how and why the intervention was effective or ineffective.

- The STOP RCT is not double blinded, and there is a risk for attrition throughout the study period.

- Due to the ongoing COVID-19 pandemic, validating self-reported smoking abstinence with carbon monoxide measures in exhaled air may not always be possible, and this might increase the risk of bias.

been proven effective in increasing the rate of successful smoking cessation attempts in the general population. ${ }^{12}$ However, the effectiveness of such smoking cessation aids in disadvantaged smokers remains unclear. More specifically, even if some web-based and behavioural interventions have demonstrated their feasibility and showed promise, we have very limited evidence on effective smoking cessation strategies for highly disadvantaged smokers. ${ }^{34}$

\section{Social inequality in smoking cessation}

The prevalence of smoking among individuals with low socioeconomic position (SEP) has remained persistently high compared with the general population in high-income countries, despite a significant drop in overall smoking prevalence over the last two decades. ${ }^{56}$ In France, despite a recent historical decrease in tobacco smoking prevalence across all social classes, the prevalence of daily 
smokers among unemployed individuals was about twice that in the active population in $2018(39.9 \%$ vs $19.5 \%){ }^{7}$

An earlier tobacco initiation, a higher cigarette consumption as well as lower quitting rates have been reported among socially disadvantaged populations. ${ }^{8} 9$ Actually, several studies have highlighted comparable rates of quitting attempts but lower successful smoking cessation rates among smokers with low SEP compared with the general population of smokers. ${ }^{10}$ Such observations advocate for devising targeted interventions addressing specific factors associated with tobacco quit attempts and successful quitting in individuals with low SEP.

Smokers with low SEP are reported to have higher nicotine dependence. ${ }^{11}$ Therefore, access and price of NRTs - which are not always eligible for public reimbursement-could be a barrier to successful smoking cessation in individuals with limited financial resources. ${ }^{12}$ In France, even if some NRT products are currently partially refunded by the French national health insurance system, ${ }^{13}$ smokers with no top-up covering insurance still have to pay at least $35 \%$ of the fees for NRT. Moreover, recent data from the French Health Barometer indicate that smokers with high SEP are more likely to quit and/ or use e-cigarettes and NRT to quit smoking compared with less socially advantaged smokers. ${ }^{14}$ Smokers with low SEP might be discouraged from using these cessation aids if they have to pay for them (even partly). ${ }^{15}$ They may also have less knowledge about the benefits and use of smoking cessation medications and e-cigarette. ${ }^{16}$

\section{Patients' preference}

The personalisation of existing treatments, based on the characteristics of individual smokers and their preference, may be more effective than standardised smoking cessation procedures. There is evidence that patients' beliefs and expectations contribute to the effects of care, and these perceptions can either enhance or reduce the effect of an intervention and its outcomes. ${ }^{17}$ In a qualitative study among smokers with low SEP, participants expressed a strong preference for a personalised quit support. ${ }^{15}$ Therefore, enabling smokers to choose their nicotine replacement product(s) based on their preference, experience and clinician advice might increase the odds of smoking cessation. Such a strategy may reveal itself to be especially effective for smokers who experience socioeconomic difficulties. Moreover, when it comes to lifestyle modification, encouraging the patient to participate in his or her medical decision-making processes might be closer to real-life situations than simply recommending one type of treatment. In fact, shared decisionmaking is positively perceived by clinicians. ${ }^{18}$

These considerations led us to develop 'STOP' (Sevrage Tabagique à l'aide d'Outils dédiés selon la Préférence: Smoking cessation using preference-based tools), a preferencebased smoking cessation intervention for smokers with low SEP. The present paper describes the protocol and design of a pragmatic, multicentre randomised controlled trial (RCT) evaluating the effectiveness of the STOP intervention.

\section{THE STOP INTERVENTION}

The STOP intervention is a heath professional-led intervention assisting smokers with low SEP in their smoking cessation attempt. It consists of routine care supplemented with free delivery of any or several type(s) of NRT (patches, inhalers, gum, tablets, etc) and/or an e-cigarette with a provision of different flavours of nicotine liquids. The individual delivery of those smoking aids is based on the corresponding smoker's preference and choice. The feasibility of the STOP intervention has been confirmed in a previous pilot study. ${ }^{19}$

\section{Patient and public involvement}

In the pilot study, a small sample of participants were asked about ways to improve the design of future research interventions in qualitative interviews. ${ }^{19}$ Their feedback was used to improve the design of this RCT.

\section{Study design and main objective}

STOP is a randomised, multicentre, single-blinded, intent-to-treat, pragmatic trial. Figure 1 illustrates the study design.

The main objective of this trial is to examine the effectiveness of the STOP intervention in real-life settings.

\section{Participating centres}

We recruited physicians to our study by sending out invitations via the newsletters of two medical societies: the 'SFTG' (Société de Formation Thérapeutique du Généraliste: Society for Therapeutic Training of the General Practitioner) and the 'SFT' (Société Francophone de Tabacologie, the French-speaking Society of Tabacology). Following these invitations, around 20 physicians expressed interest in participating in our study, clustered in around 15 centres.

Participants will be recruited by physicians and assigned to receive either the intervention or the usual care with an allocation ratio of 1:1. Participating centres include primary care practices (community, municipal or general health clinics $(\mathrm{n}=8)$ ) and public hospital-based facilities and/or healthcare institutions specialised in addiction treatment $(n=7)$. In France, people with low SEP are more likely to visit community and municipal health clinics or public hospitals, where there are generally no out-ofpocket expenses (compared with private practices).

\section{Inclusion and exclusion criteria}

To be eligible for inclusion in our trial, individuals have to be adults (aged $\geq 18$ years) who smoke at least five cigarettes per day, have at least one criterion of low SEP and are willing to lower their smoking consumption or quit smoking. Participants also have to be available for follow-up for at least 6 months after inclusion and willing to participate in our study. The five cigarettes per day limit was chosen as a criterion because it was the minimum 


\section{Pre-inclusion questionnaire, informed consent}

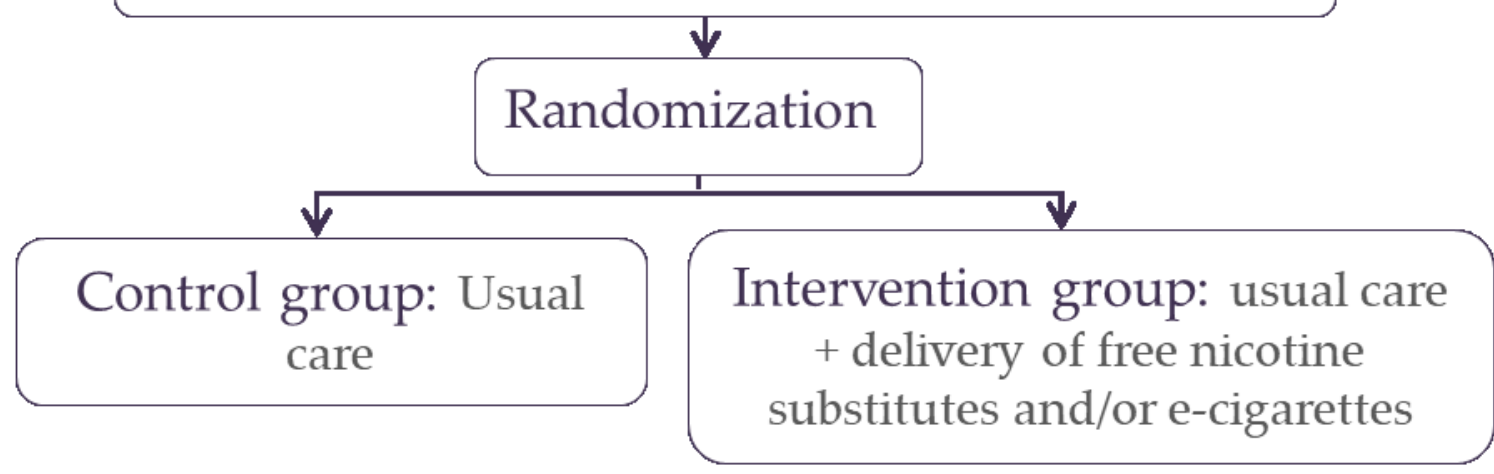

Follow-up

$\mathrm{t}=7$ to 14 days

$\mathrm{t}=1$ month

$\mathrm{t}=3$ months

$\mathrm{t}=6$ months

\section{Outcomes}

\section{-Number of cigarettes smoked} -Number of days without smoking -Smoking cessation verified by exhaled carbon monoxide (CO) levels

Figure 1 Study design of the STOP pragmatic, multicentre randomised controlled trial.

number of cigarettes smoked by participants at inclusion in the pilot feasibility study. ${ }^{19}$

The criteria of low SEP are:

- Unemployment (self-reported).

- Benefiting from at least one social assistance programme reserved for low-income individuals in France such as: the French universal health coverage (PUMA, Protection Universelle MAladie) and/or complémentaire solidaire which completes universal health coverage for care or medication, medical insurance for undocumented immigrants (AME, Aide Médicale de l'État), the disabled adult's allowance (AAH, Allocation aux Adultes Handicapés), the minimal income social support (RSA, Revenu de Solidarité Active - ASS, Allocation de Solidarité Spécifique), the family support allowance (ASF, Allocation de Soutien Familial), the family supplement (CF, Complément Familial) or a disability pension.

These criteria were chosen since they characterise individuals with a low income and are readily discernible by health professionals, therefore avoiding potentially stigmatising questions.
Exclusion criteria include physical or mental disability preventing an individual from clearly understanding or carrying out the study protocol, and being under the legal capacity of someone else (having a legally mandated surrogate). Other exclusion criteria include the inability to communicate and/or provide written informed consent in French, pregnancy, an ongoing smoking cessation therapy (pharmacotherapy including NRT or active involvement in a smoking cessation programme), current use (but not past use) of e-cigarettes and participation in another clinical study.

\section{Recruitment}

Participants will be recruited by physicians in the different study centres starting March 2021. Physicians are therefore asked to present the study to their patients who might be eligible to participate in the study. Posters and flyers inviting smokers who wish to quit to talk to their physician about smoking cessation are also supplied to participating centres.

Physicians can also carry out a preinclusion questionnaire over the phone or during a remote consultation, 
prior to a face-to-face appointment. However, study presentation, recruitment and baseline measures will only be possible for patients physically present at the study centre.

In some centres (depending on declared need), a research assistant (RA) might help physicians in describing the project to each potential participant. After a presentation of the study objective, a written informed consent (online supplemental material) will be asked from eligible patients before proceeding with randomisation.

The recruitment period in each centre will last for up to 1 year, while each participant will be followed for 6 months. However, not all centres will begin recruiting at the same time, and data collection is therefore expected to last until the end of the year 2023.

\section{Main outcome and sample size}

The main outcome measure of this study will be the 7-day point prevalence of tobacco abstinence at 6 months after inclusion (yes/no), defined as self-reported continuous abstinence for at least 7 days. This self-reported abstinence will be validated by measured exhaled carbon monoxide (CO), ${ }^{20}$ unless this measurement is unavailable due to the ongoing COVID-19 pandemic.

Smoking cessation success rates are around $3 \%-5 \%,{ }^{21}$ without the help of health professionals who usually slightly increase the chance of success (by 1\%-2\%) at 1 year. ${ }^{22}$ Therefore, adopting a conservative perspective on smoking cessation rates, we hypothesised a smoking cessation rate of $6 \%$ at 6 months in the control group (group C). ${ }^{3}$ Based on another meta-analysis, ${ }^{23}$ and also on results from our pilot study, we assumed a success rate of $14 \%$ in the intervention group (group I). The experimental plan corresponds to the comparison of two proportions: the proportion of persons who are expected to stop smoking in the control group, $\mathrm{p}_{\mathrm{C}}$, and the proportion of persons who are expected to stop smoking in the intervention group, $\mathrm{p}_{\mathrm{I}}$, with a null hypothesis $(\mathrm{H} 0)$ corresponding to $\mathrm{p}_{\mathrm{C}}=\mathrm{p}_{\mathrm{I}}$, and an alternative hypothesis $(\mathrm{H} 1)$ corresponding to $\mathrm{p}_{\mathrm{C}} \neq \mathrm{p}_{\mathrm{I}}$.

Assuming an equal number of participants in the two groups $\left(\mathrm{n}_{\mathrm{C}}=\mathrm{n}_{\mathrm{I}}\right)$, a type I error at $5 \%$ and a power at $80 \%$, 220 participants per group $(\mathrm{n}=440)$ would be needed to reject $\mathrm{H} 0$. Hypothesising the participation of 15 centres, this would correspond to around 14 participants per group and per centre. To account for potential dropouts, the experimental design eventually planned a total of 528 (440+20\% lost to follow-up) participants to be enrolled in the trial.

\section{Secondary outcomes}

The following secondary outcome measures will be assessed for all participants:

- Total number of days of abstinence (sum of the number of smoke-free days throughout the follow-up period) at 6 months after inclusion.
- Seven-day point prevalence of tobacco abstinence (Yes/No) at 1 and 3 months.

- Number of relapses.

In addition, the following measures will be collected from participants who did not stop smoking:

- The number of cigarettes smoked per day.

- The proportion of participants who have significantly reduced daily smoking (defined as a reduction in consumption by at least $50 \%$ in terms of the number of cigarettes smoked per day).

\section{Randomisation and blinding}

Each investigator will be provided with a tablet allowing access to a web-based password-protected randomisation module and an electronic case report form (eCRF) interface. Randomisation will be investigator stratified, which is thought to have a minimal effect on statistical power. ${ }^{24}$ This software is supplied by a company specialised in clinical research and will be compliant with the latest European and French regulations regarding the protection of personal data.

After verification of inclusion criteria and informed consent, the module will randomise the participant to either the intervention or the control group (1:1), and will simultaneously notify the coordinator of the study by email. To minimise selection bias, no substitution or change of group will be permitted.

Participants will be blinded to their randomisation group: participants will not know that those randomised in the intervention group will receive free e-cigarettes and/or NRT. However, all participants—as part of the informed consent statement and the study presentation-will be informed that investigators are studying how to help smokers with their quit attempts.

Physicians-who will carry out the intervention and most of the follow-up assessments-will not be blinded to treatment randomisation.

\section{The control group: usual care}

Participants randomised to the control group will be given standard care in assisting their smoking cessation attempt but without free access to NRT or e-cigarettes. Standard care depends on each health professional habitual practice; it includes motivational interviewing, advice to quit and prescription of NRTs. Health professionals will also be in a position to prescribe other treatments (eg, varenicline which is covered at $65 \%$ by the French health universal insurance and might be completely covered by a complementary health insurance-or bupropion which has to be paid completely). An investigator could also give advice on e-cigarette use if he or she finds it suitable.

At the end of the follow-up period, participants randomised to the control group will be offered an e-cigarette+e-liquid and/or NRT in sufficient quantity for 1 month, if needed and desired.

\section{The intervention}

During the face-to-face baseline appointment, participants randomised to the intervention group will receive 
the same routine care smoking cessation advice, drug prescription and support as participants in the control group. Further, participants will also have a choice of being given free NRT (transdermal patch, gum, spray, inhaler, sublingual tablets/lozenges) and/or e-cigarettes on the spot to aid with their quit attempt. E-cigarettes would be provided with e-liquids with different nicotine levels $(3,6,12$ and $16 \mathrm{mg} / \mathrm{mL}$ ) and different flavours (tobacco, mint and fruit). The provided e-cigarette is the 'Zlide Tube' (Shenzhen Innokin Technology, Shenzhen, China), an easy-to-use e-cigarette with a 3000 $\mathrm{mAh}$ rechargeable battery, a $4 \mathrm{~mL}$ tank with a sliding top refill system, provided with several spare coils, and a wall charger. E-cigarette models with refillable tanks are the most commonly used models in France, where the use of nicotine salt pod-based models is very limited.

A brief and clear description of each smoking cessation aid will be given to each participant and depending on his/her choice. At each appointment (baseline and subsequent follow-up) depending on his/her choice, he/ she will be provided with a sufficient quantity to last until the following appointment. The intervention process will be guided by the eCRF which will remind investigators to list all available products, and ask them to fill in the type and quantity of the delivered tools for participants in the intervention group.

Short didactic videos explaining how to use different NRTs and e-cigarettes will be available for participants in the intervention group (a mobile-friendly link to the video will be given), as well as for physicians accompanying participants.

During follow-up, participants in the intervention group can also be given other smoking cessation tools than the one(s) previously delivered, according to their preference and after seeing their physician (they can be given NRTs at the second appointment if they initially chose e-cigarette only and vice versa or the type of given NRTs can be changed or they can receive NRT in addition to an e-cigarette). Follow-up measures after the first (baseline) appointment might take place remotely if the participants do not require any delivery of smoking cessation tools.

Each participant in both groups will be given a diary or a 'calendar postcard' (example in figure 2) on which he/

Mois : I_l_l

Année : 202|_|

Identifiant : |_|_|-|_|_|_|

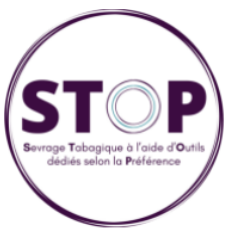

\begin{tabular}{|c|c|c|c|c|c|c|c|c|c|}
\hline & \multicolumn{2}{|l|}{1} & 2 & 3 & 4 & \multicolumn{2}{|l|}{5} & 6 & 7 \\
\hline Aides utilisées & \multicolumn{2}{|c|}{ E P G S I C } & E P G S I C & E P G S I C & E P G S I C & \multicolumn{2}{|c|}{ E P G S I C } & E P G S I C & E P G S I C \\
\hline \multicolumn{10}{|l|}{ Nb. de cigarettes } \\
\hline & \multicolumn{2}{|l|}{8} & 9 & 10 & 11 & \multicolumn{2}{|l|}{12} & 13 & 14 \\
\hline Aides utilisées & \multicolumn{2}{|c|}{ E P G S I C } & E P G S I C & E P G S I C & E P G S I C & \multicolumn{2}{|c|}{ E P G S I C } & E P G S I C & E P G S I C \\
\hline \multicolumn{10}{|l|}{$\mathrm{Nb}$. de cigarettes } \\
\hline & \multicolumn{2}{|l|}{15} & 16 & 17 & 18 & \multicolumn{2}{|l|}{19} & 20 & 21 \\
\hline Aides utilisées & \multicolumn{2}{|c|}{ E P G S I C } & E P G S I C & E P G S I C & E P G S I C & \multicolumn{2}{|c|}{ E P G S I C } & E P G S I C & E P G S I C \\
\hline \multicolumn{10}{|l|}{ Nb. de cigarettes } \\
\hline & \multicolumn{2}{|l|}{22} & 23 & 24 & 25 & \multicolumn{2}{|l|}{26} & 27 & 28 \\
\hline Aides utilisées & \multicolumn{2}{|c|}{ E P G S I C } & E P G S I C & E P G S I C & E P G S I C & \multicolumn{2}{|c|}{ E P G S I C } & \multirow[t]{2}{*}{ E P G S I C } & E P G S I C \\
\hline \multicolumn{9}{|l|}{$\mathrm{Nb}$. de cigarettes } & \\
\hline & \multicolumn{2}{|l|}{29} & 30 & 31 & \multirow{3}{*}{\multicolumn{5}{|c|}{ Pour les aides utilisées, entourez: }} \\
\hline Aides utilisées & \multirow{2}{*}{\multicolumn{2}{|c|}{ E P G S I C }} & E P G S I C & E P G S I C & & & & & \\
\hline $\mathrm{Nb}$. de cigarettes & & & & & & & & & \\
\hline \multicolumn{2}{|c|}{ 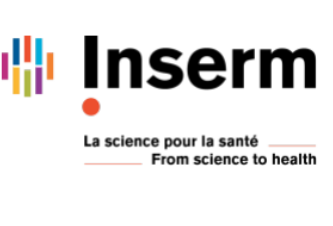 } & \multicolumn{3}{|c|}{$\begin{array}{l}\text { A la fin du mois en cours, veuillez nous } \\
\text { renvoyer cette carte grâce à l'enveloppe } \\
\text { T qui vous a été délivrée, pré-adressée } \\
\text { à: INSERM U1136, Etude STOP, bureau } \\
\text { 302, } 27 \text { rue Chaligny } 75571 \text { PARIS Cedex }\end{array}$} & \multicolumn{2}{|c|}{ E pour l'e-cigarette } & \multicolumn{2}{|c|}{$\begin{array}{l}\text { P pour les patchs } \\
\text { I pour les inhaleurs. }\end{array}$} & 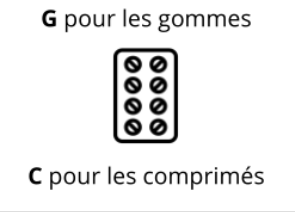 \\
\hline
\end{tabular}

Figure 2 Example of a 'calendar postcard' that will be given to each participant. 'Aides utilisées': smoking cessation aids (tools) used. 
she will document daily use of any smoking cessation aids, and the number of cigarettes smoked every day.

These postcards will be prepaid and preaddressed to return to the study investigators who would automatically fill in the corresponding data in the centralised data system.

\section{Intervention standardisation: training of medical doctors}

All participating teams (investigators) will receive individual training sessions, during which we will present to them the study protocol, the randomisation procedure and the data entry (eCRF) software.

The study protocol as well as a brief reminder of recommendations concerning the use of NRT and e-cigarettes will be presented to all participating team members.

A printed study booklet summarising the study protocol will be given out to investigators as well as a digital version on a provided tablet, as well as a video format of the training session.

Further, the fidelity of the intervention will also be assured by the structured eCRF throughout the study. The eCRF will automatically determine if participants are eligible, and will guide investigators during the intervention process.

\section{Baseline assessments}

Following randomisation, the following data will be collected from all participants:

- Demographic information, weight $(\mathrm{kg})$ and height $(\mathrm{cm})$.

- The French socioeconomical precariousness situation index (the EPICES score, Evaluation de la Précarité et des Inégalités de santé dans les Centres d'Examens de Santê). ${ }^{25}$

- Smoking status (number of cigarettes smoked daily).

- Smoking and quit attempt history.

- Other smoking-related information (the Smoking Abstinence Self-Efficacy Questionnaire, SASEQ), ${ }^{26}$ the French Tobacco Craving Questionnaire ${ }^{27}$ and nicotine dependence (Heaviness of Smoking Index ${ }^{28}$ ).

- Other substance use (cannabis, and other illicit drugs).

- Mental health (a short two-item measure: Patient Health Questionnaire-2 (PHQ-2) ${ }^{29}$ ).

- Concomitant medication use and health problems.

\section{Other follow-up measures}

Follow-up appointments will take place around 10 days, and 1, 3 and 6 months after inclusion. The first follow-up can take place between 7 and 14 days after inclusion. Additional follow-up appointments can take place on the request of the physician and/or the participant in both groups. If needed, follow-up measures can be conducted via telephone by the health professional or an RA.

At each appointment, data will be collected from all participants on:

- Weight $(\mathrm{kg})$.
- Smoking status, the number of cigarettes smoked (patients will be asked to bring their 'calendar postcards' that have not been already posted).

- Past quit attempt(s) and number of relapses, as well as positive and/or negative perceptions related to withdrawal.

- The SASEQ and mental health (PHQ-2).

- Use of any other smoking cessation pharmacological treatments.

- (If possible), exhaled CO measured electrochemically in parts per million (ppm) by a CO tester (piCO+ Smokerlyzer, Bedfont Scientific, Harrietsham, UK) with values above 6 indicating recent tobacco consumption. ${ }^{30}$

Individuals randomised in the intervention group would also be asked of their use and perception of the smoking cessation aid product(s) delivered, as well as the frequency of use and their views on using NRT and e-cigarettes as smoking cessation aids, as well as their perceptions concerning these tools.

\section{Promoting retention}

In addition to the calendar cards, other measures will be taken to promote retention. When a participant misses one of the follow-up visits scheduled in the protocol, the follow-up questionnaire may be completed remotely by the investigator (by telephone or remote consultation), or by phone by an RA. The method of follow-up (face to face or remotely) will be noted in the eCRF.

\section{Data monitoring}

Data monitoring will be carried out by two different clinical research associates, one of whom is independent of the research team.

\section{Adverse events}

The two principal investigators will have the responsibility to monitor adverse events which are systematically measured in all follow-up assessments. These events will also be monitored by two different RAs. Every adverse event will be examined by the steering committee and promptly reported to the scientific committee which will decide whether or not the study should continue.

\section{Statistical analyses}

Primary analyses will be carried out on an intention-totreat (ITT) basis. The ITT population will comprise all participants randomised, regardless of whether the intervention was actually received or whether they subsequently withdrew or deviated from the protocol.

In the main analysis, simple incidence rates and relative and absolute risks will be calculated for all binary variables, and the two groups will be compared using logbinomial regression to estimate associated relative risks (RRs) in the primary analysis.

In secondary analysis, models will be adjusted for appropriate covariates (sociodemographic characteristics and other potential confounders) to explore the impact of these covariates on the intervention effect, and taking 
into account the hierarchical structure of the data and investigator's effect (eg, a random effect at the investigator level). ${ }^{31}$

The proportion of participants who have significantly reduced their daily smoking level will be calculated and non-adjusted and adjusted RRs according to randomisation groups will also be calculated for this outcome.

The distribution of all continuous outcomes will be assessed for normality and skewed data will be subjected to an appropriate transformation prior to analysis. The change from baseline in the number of cigarettes smoked per day will be analysed using repeated measures models (mixed-effects regression models) adjusting for baseline value, and taking into account the investigator level (random effect).

Finally, if the trial results declare the intervention as successful, we will estimate the distribution of the number of days (mean, median, IQR,...) of the time (number of days) during which participants in the intervention group used smoking cessation tools, to provide decision-makers with a recommendation for how long smoking aids should be prescribed.

A per-protocol analysis will be performed for the primary outcome for which-to check the robustness of the results-participants with any major protocol violation (such as skipping appointments, withdrawal and loss to follow-up) will be excluded. Time to first smoking relapse will also be analysed using Kaplan-Meier curves, the log-rank test and Cox proportional hazards regression analyses. In case of multiple quit attempts during the follow-up period (repeated events), we will use frailty models to estimate time to event. ${ }^{32}$

All tests of statistical significance will be two tailed, and all analyses will be performed using SAS V.9.4 (or higher).

Interim statistical analysis will be carried out around 6 months after the first recruitment by the study principal investigator.

\section{Economic analysis}

The study will also include an economic evaluation. The corresponding analysis will adopt the perspective of the payer of the intervention (which would be the French health insurance system). The time horizon of the study will be the 6-month follow-up and therefore no discounting rate will be applied to cost and health outcomes. Based on such a perspective, the difference between the costs for the 6-month management of an individual in the intervention group-research-related costs will of course be excluded-versus the control group will be calculated. The cost difference will be contrasted to that of the quitters at 6 months observed in the two arms, leading to the estimation of a standard incremental cost-effectiveness ratio estimating the incremental cost of the intervention per quitter at 6 months.

Incidentally, each participant will be informed of the difference between his/her costs of tobacco consumption at baseline and 6 months.
ETHICS AND DISSEMINATION

\section{Ethical approval}

The STOP RCT was approved by the 'Île de France II' Institutional Review Board on 8 September 2020 (CPP Île de France II; Ref No: 20.01.31.65528 RIPH2 HPS).

\section{Informed consent}

Prior to inclusion in our study, participants will receive a presentation of key information about the clinical trial, orally and with a written consent form. The form also contains information on data protection (only the investigator will have access to non-identifying data). Participants will also be reminded of their right to revoke their participation in the study at any moment, and to request the suppression of their data. All participants will be provided with a copy of the study presentation and consent form. All study forms have been reviewed by the ethics committee which authorised the trial.

\section{Dissemination}

Results of this study will be communicated at scientific meetings and submitted for publication in peer-reviewed journals in accordance with the Consolidated Standards of Reporting Trials statement, and Template for Intervention Description and Replication checklist. ClinicalTrials. gov record will also be updated regularly.

The principal investigators may grant access to the full protocol and to the statistical code on request.

\section{Steering committee and scientific council}

The steering committee is constituted by the two principal investigators (FEK and MM), the president of the French-speaking French Society of Tabacology (ALLF), the president of the Society of Therapeutic Training of the Generalist Practitioner (study sponsor) (GI) and the project managers and RAs. Its role is to follow the study implementation, and to implement the recommendations of the scientific committee.

The scientific committee is constituted by the two principal investigators, the study's qualitative researcher, a methodology expert and the study's economist. This council was involved in the drafting of the protocol (methodology, main outcomes, analysis, etc) and questionnaires, and will be responsible for drafting any possible amendments. Its role will be to validate the scientific orientations of the project, to oversee analysis and to guarantee its medical and scientific quality.

The two committees meet regularly as required by the study's progress, with a minimum of two meetings a year.

\section{Process evaluation}

According to the Medical Research Council, process evaluation endeavours to 'assess fidelity and quality of implementation, clarify causal mechanisms and identify contextual factors associated with variation in outcomes' ${ }^{33}$ In a nutshell, it is a systematic process implemented to understand what an intervention or a programme does and how well it does it. There is evidence that in health 
promotion and prevention programmes, the quality of implementation affects the outcomes obtained. ${ }^{34}$

This is why we will be tracking and documenting all activities and outputs of the STOP intervention. Throughout the study, we will record: the amount of money spent, the number of investigators, RA, and other researchers associated or students involved in the intervention, the number and timing of smoking cessation tools delivered for each investigator and the timing of each training session per recruited investigator.

We will also prospectively describe the characteristics of the intervention, its context and timeline. This description will be important for understanding and interpreting the results. In each study centre, the average ratio between the number of recruited participants and the number of screened smokers as well as questionnaire completion rates will be estimated.

We will also collect regular qualitative and quantitative data on trial activities, and participants and investigators' perception of the study intervention. Researchers specialised in qualitative studies will carry out semistructured interviews (in person or via telephone) with participating health professionals and participants, during and after the intervention. During the interviews, investigators and participants will be asked about perceived barriers and facilitators to the implementation of the intervention, and their experiences with implementing the intervention under routine conditions, organisational matters (time, logistic aspects, randomisation, etc) as well as the perceived advantages of the intervention. In addition, physicians $(n=3-5)$ will be asked about their experiences motivating patients to quit smoking.

Among participating smokers $(n=10-12)$, we will ensure that interviews are conducted with participants who completed the full protocol as well as participants who dropped out if possible.

\section{IMPLICATIONS}

We expect that the above-detailed pragmatic intervention, which is embedded in the healthcare system, will provide a 'real-world effectiveness' evidence of a scalable smoking cessation intervention which could contribute to the reduction of health inequalities.

\section{Trial sponsor}

The trial sponsor is "the SFTG-Recherche" (Société de Formation Thérapeutique du GénéralisteRecherche : Society for Therapeutic Training of the General Practitioner), Society forTherapeutic Training of the General Practitioner.sftg-recherche.fr/

\section{Author affiliations}

${ }^{1}$ Sorbonne Université, INSERM UMR_S 1136, Institut Pierre Louis d'Épidémiologie et de Santé Publique (IPLESP), department of Social epidemiology, Paris, France

${ }^{2}$ Eepidemiology department, University Hospital Group of Psychiatry and Neurosciences (GHU Paris), Paris, France

${ }^{3}$ Sorbonne Université, INSERM UMR_S 1136, Institut Pierre Louis d'Épidémiologie et de Santé Publique (IPLESP), CLEPIVIR research team, Paris, France
${ }^{4}$ CSAPA Charonne, Paris, France

${ }^{5}$ Addiction Centre, Hôpital Européen Georges Pompidou, Pôle PsychiatrieAddictologie, Hôpitaux Universitaires Paris-Ouest, Paris, France

${ }^{6}$ Network of research in substance use disorder, Fédération hospitalo-universitaire, Paris, France

${ }^{7}$ INSERM, Institut Pierre Louis d'Epidémiologie et de Santé Publique (IPLESP), Sorbonne University Faculty of Medicine, Paris, France

${ }^{8}$ SFTG Recherche (Société de Formation Thérapeutique du Généraliste Recherche), Paris, France

Contributors The study concept and design were conceived by FEK and MM. FEK, MM, MH, BM, GI, GH, TEA and ALLF contributed to the development and implementation of the study protocol. The protocol writing was directed and critically reviewed by all coauthors.

Funding This work was supported by a grant from the French National Cancer Institute (INCA). Award/grant number: IRESP-AAP TABAC 2018.

Disclaimer INCA had no role in study design and the decision to submit the protocol for publication.

Competing interests None declared.

Patient consent for publication Not required.

Provenance and peer review Not commissioned; externally peer reviewed.

Supplemental material This content has been supplied by the author(s). It has not been vetted by BMJ Publishing Group Limited (BMJ) and may not have been peer-reviewed. Any opinions or recommendations discussed are solely those of the author(s) and are not endorsed by BMJ. BMJ disclaims all liability and responsibility arising from any reliance placed on the content. Where the content includes any translated material, BMJ does not warrant the accuracy and reliability of the translations (including but not limited to local regulations, clinical guidelines, terminology, drug names and drug dosages), and is not responsible for any error and/or omissions arising from translation and adaptation or otherwise.

Open access This is an open access article distributed in accordance with the Creative Commons Attribution Non Commercial (CC BY-NC 4.0) license, which permits others to distribute, remix, adapt, build upon this work non-commercially, and license their derivative works on different terms, provided the original work is properly cited, appropriate credit is given, any changes made indicated, and the use is non-commercial. See: http://creativecommons.org/licenses/by-nc/4.0/.

\section{ORCID iDs}

Fabienne El-Khoury http://orcid.org/0000-0002-6915-6850

Gladys Ibanez http://orcid.org/0000-0002-5592-699X

Maria Melchior http://orcid.org/0000-0002-2377-619X

\section{REFERENCES}

1 Stead LF, Perera R, Bullen C, et al. Nicotine replacement therapy for smoking cessation. Cochrane Database Syst Rev 2012;11:CD000146.

2 Grabovac I, Oberndorfer M, Fischer J, et al. Effectiveness of electronic cigarettes in smoking cessation: a systematic review and meta-analysis. Nicotine Tob Res 2021;23:625-34.

3 Bryant J, Bonevski B, Paul C, et al. A systematic review and metaanalysis of the effectiveness of behavioural smoking cessation interventions in selected disadvantaged groups. Addiction 2011;106:1568-85.

4 Brunette MF, Gunn W, Alvarez H, et al. A pre-post pilot study of a brief, web-based intervention to engage disadvantaged smokers into cessation treatment. Addict Sci Clin Pract 2015;10:3.

5 Kulik MC, Hoffmann R, Judge K, et al. Smoking and the potential for reduction of inequalities in mortality in Europe. Eur J Epidemiol 2013;28:959-71.

6 Agaku IT, King BA, Dube SR, et al. Current cigarette smoking among adults - United States, 2005-2012. MMWR Morb Mortal Wkly Rep 2014:63:29-34.

7 Andler R, Richard J-B, Guignard R. Baisse de la prévalence du tabagisme quotidien parmi les adultes : Résultats du baromètre de santé publique France 2018, 2019.

8 El-Khoury F, Bolse C, Melchior F. Les adolescents face au tabac : l'effet du type de scolarité suivie : données de l'étude nationale DePICT. BEH, 2018.

9 El-Khoury Lesueur F, Bolze C, Melchior M. Factors associated with successful vs. unsuccessful smoking cessation: data from a nationally representative study. Addict Behav 2018;80:110-5. 
10 Kotz D, West R. Explaining the social gradient in smoking cessation: it's not in the trying, but in the succeeding. Tob Control 2009;18:43-6.

11 Hobkirk AL, Krebs NM, Muscat JE. Income as a moderator of psychological stress and nicotine dependence among adult smokers. Addict Behav 2018;84:215-23.

12 Twardella D, Brenner $\mathrm{H}$. Effects of practitioner education, practitioner payment and reimbursement of patients' drug costs on smoking cessation in primary care: a cluster randomised trial. Tob Control 2007;16:15-21.

13 Ameli.com. Prise en charge des substituts nicotiniques. Available: https://www.ameli.fr/assure/remboursements/rembourse/ medicaments-vaccins-dispositifs-medicaux/prise-charge-substitutsnicotiniques [Accessed 25 Jun 2018].

14 Guignard R, Richard J-B, Pasquereau A. Tentatives d'arrêt du tabac au dernier trimestre 2016 et lien avec Mois sans tabac : premiers résultats observés dans le Baromètre santé 2017 // Quit attempts in the last quarter of 2016 and relation with Mois sans tabac: first results from the 2017 Health Barometer, 2018. Available: http://invs. santepubliquefrance.fr/beh/2018/14-15/2018_14-15_6.html

15 Bryant J, Bonevski B, Paul C, et al. Developing cessation interventions for the social and community service setting: a qualitative study of barriers to quitting among disadvantaged Australian smokers. BMC Public Health 2011;11:493.

16 Pokhrel P, Little MA, Fagan P, et al. Electronic cigarette use outcome expectancies among college students. Addict Behav 2014;39:1062-5.

17 Bowling A, Ebrahim S. Measuring patients' preferences for treatment and perceptions of risk. Quality and Safety in Health Care 2001;10:i2-8.

18 Pollard S, Bansback N, Bryan S. Physician attitudes toward shared decision making: a systematic review. Patient Educ Couns 2015;98:1046-57.

19 Héron M, Melchior M, El-Khoury F. Free access to nicotine substitutes and e-cigarette for tobacco cessation: stop a pilot intervention. Eur J Public Health 2019;29:451.

20 West R, Hajek P, Stead L, et al. Outcome criteria in smoking cessation trials: proposal for a common standard. Addiction 2005;100:299-303.

21 Panel TU and DG. Treating tobacco use and dependence: 2008 update. US department of health and human services, 2008.

22 Stead LF, Buitrago D, Preciado N. Physician advice for smoking cessation. Cochrane Database Syst Rev 2008;CD000165.
23 Hartmann-Boyce J, Hong B, Livingstone-Banks J, et al. Additional behavioural support as an adjunct to pharmacotherapy for smoking cessation. Cochrane Database Syst Rev 2019;6:CD009670.

24 Anisimov VV. Effects of unstratified and centre-stratified randomization in multi-centre clinical trials. Pharm Stat 2011;10:50-9.

25 Sass C, Moulin J-J, Guéguen R. Le score Epices: un score individuel de précarité. Construction Du score et mesure des relations avec des données de santé, dans une population de 197389 personnes. Bull Épidémiologique Hebd 2006;14:93-6.

26 Spek V, Lemmens F, Chatrou M, et al. Development of a smoking abstinence self-efficacy questionnaire. Int J Behav Med 2013;20:444-9.

27 Berlin I, Singleton EG, Heishman SJ. Validity of the 12-Item French version of the tobacco craving questionnaire in treatment-seeking smokers. Nicotine Tob Res 2010;12:500-7.

28 Borland $\mathrm{R}$, Yong $\mathrm{H}-\mathrm{H}, \mathrm{O}^{\prime}$ Connor RJ, et al. The reliability and predictive validity of the Heaviness of smoking index and its two components: findings from the International tobacco control four country study. Nicotine Tob Res 2010;12 Suppl:S45-50.

29 Kroenke K, Spitzer RL, Williams JBW. The patient health Questionnaire-2: validity of a two-item depression screener. Med Care 2003;41:1284-92.

30 Bedfont Scientific Ltd. Smokerlyzer®Range for use with piCO, piCObaby and Micro+, 2018. Available: https://www.bedfont.com/ documents/2910-LAB679\%20Smokerlyser\%20Manual\%20-\% 20lssue\%204.pdf

31 Brown J, Michie S, Geraghty AWA, et al. Internet-Based intervention for smoking cessation (StopAdvisor) in people with low and high socioeconomic status: a randomised controlled trial. Lancet Respir Med 2014:2:997-1006.

32 Amrhein J. Introduction to frailty models. Available: http://support. sas.com/resources/papers/proceedings14/1492-2014.pdf [Accessed 23 Dec 2014].

33 Moore GF, Audrey S, Barker M, et al. Process evaluation of complex interventions: medical Research Council guidance. BMJ 2015;350:h1258

34 Durlak JA, DuPre EP. Implementation matters: a review of research on the influence of implementation on program outcomes and the factors affecting implementation. Am J Community Psychol 2008;41:327-50. 